\title{
Maintenance in myeloma patients achieving complete response after upfront therapy: a pooled analysis
}

\author{
Chiara Cerrato ${ }^{1}$. Francesco Di Raimondo ${ }^{2}$ - Lorenzo De Paoli ${ }^{3}$. Stefano Spada ${ }^{1}$. Francesca Patriarca ${ }^{4}$. \\ Claudia Crippa ${ }^{5} \cdot$ Roberto Mina $^{1} \cdot$ Tommasina Guglielmelli $^{6}$. Dina Ben-Yehuda ${ }^{7}$. Daniela Oddolo ${ }^{1}$. Chiara Nozzoli ${ }^{8}$. \\ Emanuele Angelucci ${ }^{9}$. Nicola Cascavilla ${ }^{10}$ - Rita Rizzi $^{11}$ - Stefano Rocco ${ }^{12}$. Luca Baldini $^{13}$. Elena Ponticelli ${ }^{1}$. \\ Magda Marcatti ${ }^{14}$. Clotilde Cangialosi ${ }^{15} \cdot$ Tommaso Caravita $^{16}$. Giulia Benevolo ${ }^{17}$. Roberto Ria ${ }^{18}$. Arnon Nagler ${ }^{19}$. \\ Pellegrino Musto ${ }^{20} \cdot$ Paola Tacchetti ${ }^{21} \cdot$ Paolo Corradini $^{22} \cdot$ Massimo Offidani $^{23} \cdot$ Antonio Palumbo $^{1,25}$. \\ Maria Teresa Petrucci ${ }^{24} \cdot$ Mario Boccadoro ${ }^{1} \cdot$ Francesca Gay $^{1}(\mathbb{0}$
}

Received: 27 December 2017 / Accepted: 13 April 2018

(c) Springer-Verlag GmbH Germany, part of Springer Nature 2018

\begin{abstract}
Purpose Maintenance demonstrated to improve survival in newly diagnosed multiple myeloma (NDMM) patients and the achievement of complete response (CR) is a strong predictor of survival. Nevertheless, the role of maintenance according to response after induction/consolidation has not been investigated so far. To evaluate the impact of maintenance according to response, we pooled together and retrospectively analyzed data from 955 NDMM patients enrolled in two trials (GIMEMAMM-03-05 and RV-MM-PI-209).

Methods Primary endpoints were progression-free survival (PFS)1, PFS2 and overall survival (OS) of CR patients randomized to maintenance and no maintenance. Secondary endpoints were PFS1, PFS2 and OS in very good partial response/ partial response (VGPR/PR) patients.

Results Overall, 213 patients obtained CR after induction/consolidation, 118 received maintenance and 95 no maintenance. In patients achieving CR, maintenance significantly improved PFS1 (HR 0.50, $P<0.001$ ), PFS2 (HR 0.58, P 0.02) and OS (HR 0.51, P 0.02) compared with no maintenance; the advantage was maintained across all the analyzed subgroups according to age, International Staging System (ISS) stage, cytogenetic profile and treatment. Similar features were seen in VGPR/ PR patients.

Conclusion Maintenance prolonged survival in CR and in VGPR/PR patients. The benefit in CR patients suggests the importance of continuing treatment in patients with chemo-sensitive disease.

Trial registration The two source studies are registered at ClinicalTrials.gov: identification numbers NCT01063179 and NCT00551928.
\end{abstract}

Keywords Multiple myeloma (MM) $\cdot$ Maintenance therapy $\cdot$ Newly diagnosed $\cdot$ Complete response $(\mathrm{CR}) \cdot$ Prognosis

\section{Introduction}

Multiple myeloma (MM) is an incurable plasma cell neoplasm that accounts for approximately $13 \%$ of hematologic cancers (Altekruse et al. 2010). The standard of care for MM

Electronic supplementary material The online version of this article (https://doi.org/10.1007/s00432-018-2641-5) contains supplementary material, which is available to authorized users.

Francesca Gay

fgay@cittadellasalute.to.it

Extended author information available on the last page of the article patients ineligible for autologous stem cell transplantation (ASCT) is the combination of melphalan-prednisone (MP) plus bortezomib (VMP) or thalidomide (MPT) or the association of lenalidomide and low-dose dexamethasone (Rd) (San Miguel et al. 2008; Mateos et al. 2010; Fayers et al. 2011; Benboubker et al. 2014). A progression-free survival (PFS) advantage with bortezomib in combination with Rd in comparison with $\mathrm{Rd}$ alone in patients who are not candidates to ASCT has recently been demonstrated (Durie et al. 2017).

In patients eligible for ASCT, the standard approach consists of a novel agent-based induction regimen (proteasome inhibitor and/or immunomodulatory drug) followed by 
high-dose melphalan and ASCT (MEL200-ASCT) (Moreau et al. 2015).

Recent data clarified the role of maintenance therapy (Palumbo and Anderson 2011; Palumbo et al. 2015). In patients ineligible for ASCT, continuous therapy with lenalidomide and dexamethasone improved PFS and overall survival (OS) as compared with MPT (Benboubker et al. 2014). A consistent survival advantage was also observed in patients treated with bortezomib-thalidomide (VT) maintenance (Palumbo et al. 2010, 2014a; Mateos et al. 2012). In patients eligible for ASCT, four different trials demonstrated the PFS advantage of lenalidomide maintenance (Attal et al. 2012; McCarthy et al. 2012; Palumbo et al. 2014b; Jackson et al. 2016a), while a recent meta-analysis has shown also a significant OS benefit (Attal et al. 2016).

The association between depth of response and survival had been highlighted in many studies (van de Velde et al. 2007). So far, only one randomized trial (Myeloma XI) investigated the role of a response-adapted approach. Preliminary results showed a response upgrade in around $40 \%$ of patients and a significant PFS improvement in patients achieving a suboptimal response after IMIDs who received sequential treatment with bortezomib-cyclophosphamidedexamethasone (VCD). On the other hand, patients who reached very good partial response/complete response (VGPR/CR) did not receive any further induction, assuming that the achievement of high-quality response may not need intensification (Jackson et al. 2016b). No trial has so far prospectively evaluated the effect of intensified treatment or prolonged treatment in patients achieving good quality responses.

We pooled together data from two phase III trials comparing maintenance vs no maintenance. The aim of our analysis was to evaluate the impact of maintenance according to response achieved after induction/consolidation therapy. As primary objective, we evaluated the effect of maintenance treatment in patients achieving high-quality response to induction/consolidation, defined as CR; as secondary objective, we analyzed the impact of maintenance in patients with suboptimal response (VGPR/PR) after induction/consolidation therapy.

\section{Materials and methods}

\section{Patients and treatment}

We selected two phase III trials (GIMEMA-MM-03-05 and RV-MM-PI-209) coordinated by the same principal investigator. In both trials, newly diagnosed MM patients received maintenance or no maintenance after induction/consolidation. Details and results of the two studies were previously published (Palumbo et al. 2010, 2014b). Additional material and methods are reported in the Supplementary Appendix.

\section{Clinical endpoints}

The primary endpoints of the analysis were PFS1, PFS2 and OS in patients eligible to maintenance or no maintenance who achieved CR after induction/consolidation. Secondary endpoints were PFS1, PFS2 and OS in patients eligible to maintenance or no maintenance who achieved a VGPR/PR after induction/consolidation (Durie et al. 2006; European Medicines Agency 2012).

PFS1, PFS2 and OS are defined in the supplementary material.

\section{Statistical analysis}

We performed a retrospective, non-preplanned analysis. Data from the two trials were pooled together and analyzed. As patients were randomized at study entry but started maintenance after a median of 10 months from randomization (that corresponds to the median time of induction/consolidation), a landmark analysis with a landmark point at 10 months from start of therapy was performed. We included patients alive and progression free after 10 months from start of treatment, and who had achieved at least a partial response (PR) (Durie et al. 2006) after induction/consolidation. Patients were stratified according to response achieved (CR vs VGPR/PR).

Time-to-event data were analyzed using the Kaplan-Meier method; treatment groups were compared with the log-rank test. The Cox proportional hazards models were used to estimate adjusted hazard ratios (HRs) and 95\% confidence intervals (CIs) for the main comparisons; Grambsch and Therneau test was used for testing the proportional hazard assumption.

To identify if the benefit of maintenance vs no maintenance varied in specific subsets of patients, subgroup analyses using interaction terms between treatment and each of the covariate included in the Cox model were performed. Patients were categorized according to age $(\leq 65,66-75$, $>75$ ), ISS Stage (I, II, III, missing data), cytogenetic risk [high (presence of at least one of the following: translocation $(4 ; 14)$, translocation $(14 ; 16)$, deletion 17$)$, standard (absence of $t(4 ; 14), t(14 ; 16)$ and deletion 17)] and trial (GIMEMAMM-03-05, RV-MM-PI-209; patients enrolled in the RVMM-PI-209 were also analyzed according to the specific pre-maintenance therapy received [MPR vs MEL200]).

All HRs were estimated with their 95\% CI and two sided $p$ values. Differences between categorical variables were examined by Fisher's exact test. Data were analyzed as of December, 2016 using R (Version 3.1.1). 


\section{Results}

\section{Patients}

Data from 955 patients enrolled in the 2 trials were pooled together; 550 patients were eligible for maintenance. A total of 213 patients achieved a CR after induction/consolidation, 118 of them were randomized to maintenance and 95 to no maintenance. Three-hundred and sixteen patients achieved a VGPR/PR after induction/consolidation, 151 of them received maintenance treatment and $165 \mathrm{did}$ not (Figure S1). Of 269 patients receiving maintenance, 120 discontinued within two years of therapy. The main reasons for discontinuation were PD (66\%), toxicity $(18 \%)$ and patients' or medical decision (16\%). Of note, rate of discontinuation of maintenance was higher in patients older than 75 years vs younger (68 vs $42 \%$; $P=0.037$ ).

Patient demographics and disease characteristics in CR and VGPR/PR patients randomized to maintenance and no maintenance were well-balanced (Table S1). High-risk patients (ISS stage III and high-risk cytogenetic profile) were equally distributed in the maintenance and no maintenance groups.

\section{CR population}

The median follow-up from landmark point was 56 months. The median PFS1 was 47 months for the maintenance group vs 28 months for the no maintenance group (HR 0.50, 95\% CI 0.35-0.71, P<0.001) (Fig. 1a). The PFS1 benefit associated with maintenance therapy was consistent in the different subgroups, although it was more pronounced in patients $\leq 65$ years (HR 0.26) as compared with those aged 66-75 years (HR 0.65) and $>75$ years (HR 0.89) ( $P=0.04$ for interaction). In accordance, a more pronounced advantage in the RV-MM-PI-209 trial where only patients $\leq 65$ years were enrolled was noticed. No other differences were detected between the different analyzed subgroups (Fig. 1b).

The median PFS2 was not reached in the maintenance group and was 57 months in the no maintenance group (HR $0.58,95 \%$ CI $0.37-0.91, P=0.02$ ), suggesting a longterm survival benefit associated with maintenance treatment (Fig. 2a). The PFS2 benefit with maintenance was consistent in all the different analyzed subgroups (Fig. 2b).

The 5-year OS was 82 vs $66 \%$ (median not reached in both groups), respectively, in patients who received maintenance compared with those who did not (HR 0.51, 95\% CI 0.29-0.91, $P=0.02$ ), (Fig. 3a). The OS advantage was confirmed among all analyzed subgroups, but it was less evident in patients with ISS stage I (HR 0.97) (Fig. 3b).
This analysis is, however, limited by the low number of events so far in this good prognosis group.

\section{VGPR/PR population}

The median follow-up from landmark point was 44 months. Of 151,11 patients increased their best response during maintenance treatment. The median PFS1 was 28 months in the maintenance group vs 18 months in the no maintenance group (HR $0.55,95 \%$ CI $0.42-0.72, P<0.001$ ) (Figure S2 Panel A). Similarly to CR patients, the PFS1 benefit associated with maintenance therapy was consistent in all the analyzed subgroups, and it was more evident in patients $\leq 65$ years (HR 0.5) and aged 66-75 (HR 0.54) as compared with those aged $>75$ years (HR 0.86) (Fig. S2 Panel B).

The median PFS2 was 53 months vs 41 months in patients randomized to maintenance vs no maintenance (HR 0.64, 95\% CI 0.46-0.89, $P=0.01$ ) (Fig. S3 Panel A). No difference was observed in the subgroups analysis, but the advantage was less evident again in patients aged $>75$ years $(\mathrm{HR}$ 0.78) (Fig. S3 Panel B).

The median OS was not reached in the two groups, and the 5-year OS was 63 vs $57 \%$ (HR 0.75, 95\% CI 0.50-1.12, $P=0.16$ ) (Fig. S4 Panel A). The advantage was again more evident in patients aged $\leq 65$ years (HR 0.56) in comparison with those aged 66-75 years (HR 0.87) or $>75$ years (HR 0.85) (Fig. S4 Panel B).

Interestingly, the highest survival rates (in terms of PFS1, PFS2 and OS) were noticed in CR patients receiving maintenance; survival of patients achieving a CR who did not receive maintenance was similar to the one of VGPR/PR patients treated with maintenance (Fig. $4 a-c)$.

\section{Discussion}

Previous reports demonstrated that maintenance treatment was associated not only with a PFS, but in some studies also with an OS advantage (Fayers et al. 2011; Attal et al. 2012, 2016; McCarthy et al. 2012; Palumbo et al. 2014a, b, 2015; Benboubker et al. 2014; Jackson et al. 2016a), and nowadays a growing number of clinical studies are including maintenance as part of the therapeutic approach. The impact on survival of achieving a CR in comparison with VGPR/PR is well-known (van de Velde et al. 2007) and the value of prolonging treatment in patients with suboptimal response (VGPR/PR) is commonly recognized and accepted. Nevertheless, whether therapy should be continued in patients achieving CR remains an open issue. To the best of our knowledge, this pooled analysis is the first study to investigate the effect of a maintenance therapy in MM 
Fig. 1 Progression-free survival 1 according to maintenance/ no maintenance in $\mathrm{CR}$ patients: $\mathbf{a}$ analysis in all patients; $\mathbf{b}$ subgroup analysis

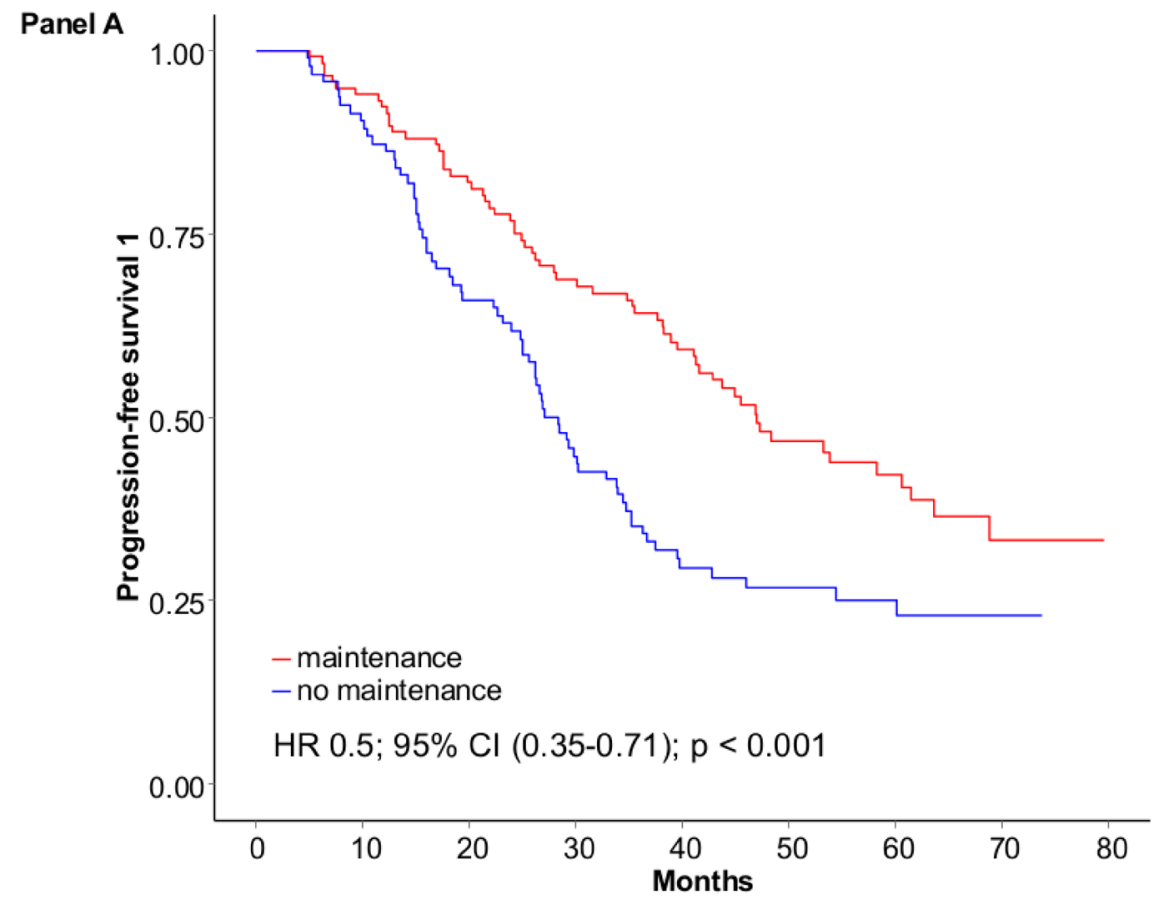

Panel B

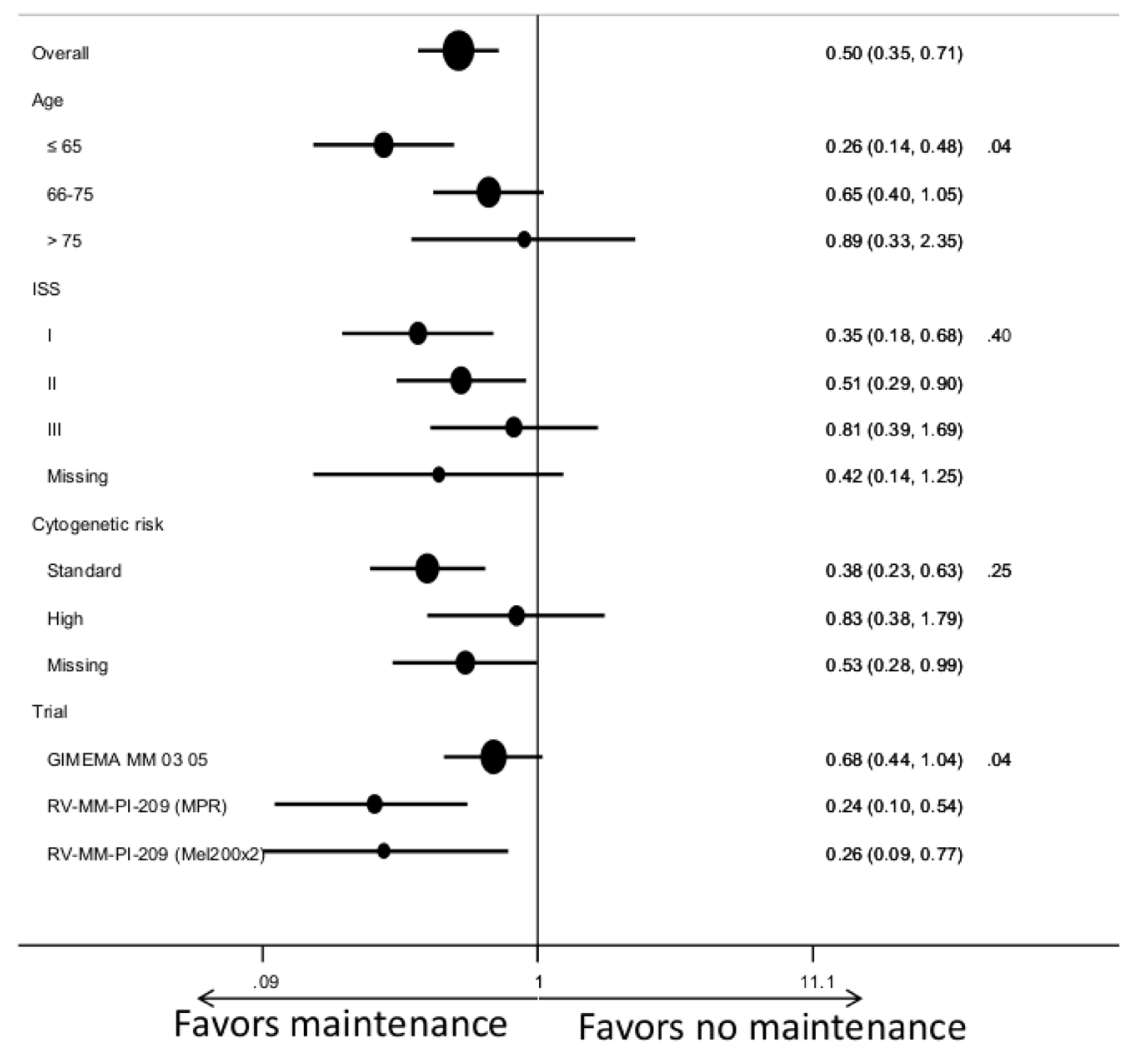

patients according to the response achieved after induction/ consolidation.

Prolonged treatment in patients with suboptimal response to therapy improved PFS1, PFS2 and OS, as expected (Figs. S2, S3, S4). Of note, maintenance therapy significantly improved PFS1 (5-year PFS1: 42 vs 25\%, HR 0.50, $P<0.001$ ), PFS2 (5-year PFS2: 62 vs $45 \%$, HR $0.58, P=0.02)$ and OS (5-year OS: 82 vs $66 \%$, HR 0.51 , 
Fig. 2 Progression-free survival 2 according to maintenance/ no maintenance in $\mathrm{CR}$ patients: $\mathbf{a}$ analysis in all patients; $\mathbf{b}$ subgroup analysis

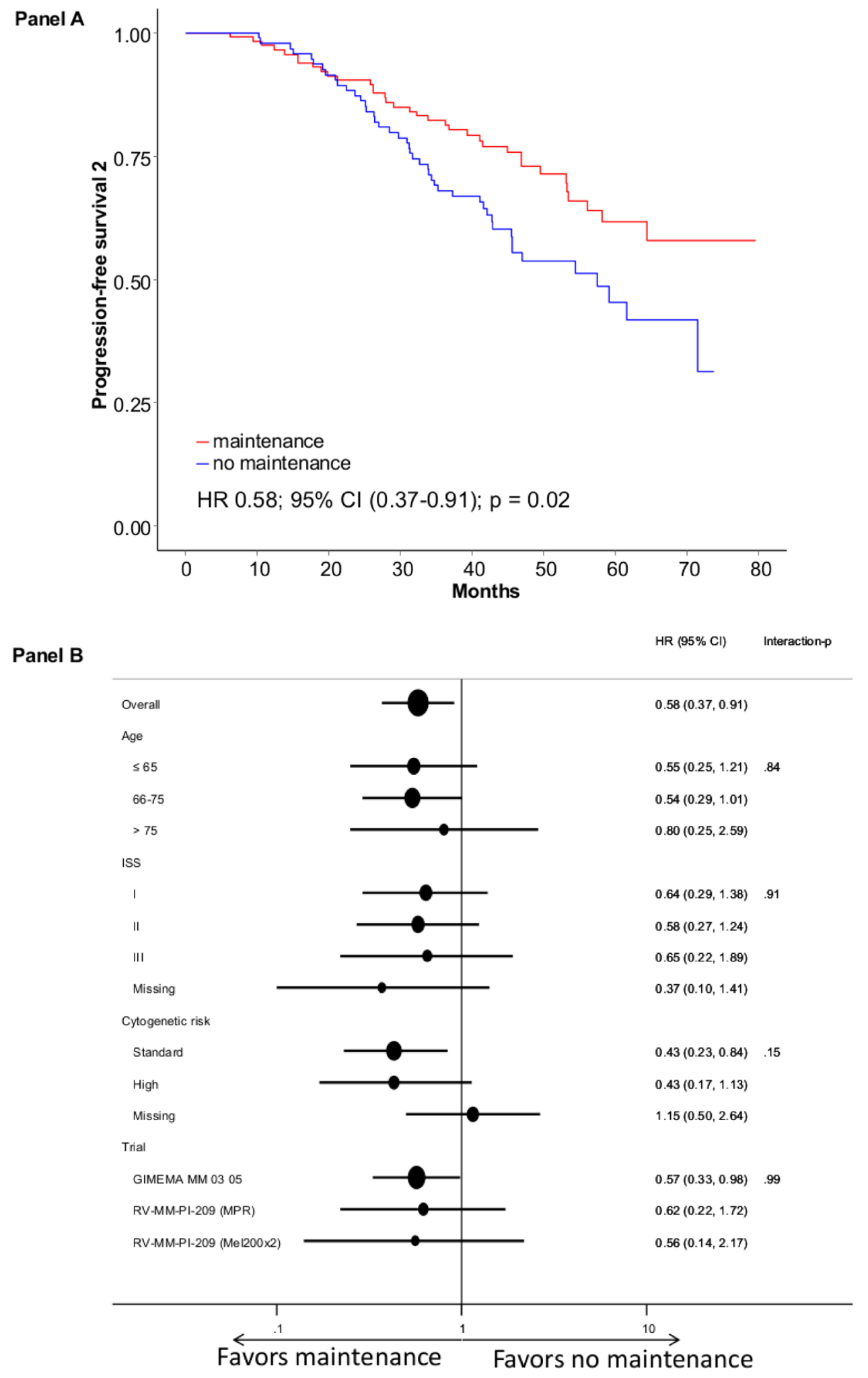

$P=0.02)$ also in patients who achieved a CR after induction/consolidation (Figs. 1, 2, 3). Within both response categories (CR and VGPR/PR populations), this benefit could depend on the ability of maintaining the best response achieved rather than on increasing response during maintenance therapy. In fact, only around $7 \%$ of VGPR/PR patients increased their response during maintenance. Unfortunately, no data on MRD evaluation were 
Fig. 3 Overall survival according to maintenance/ no maintenance in CR patients: $\mathbf{a}$ analysis in all patients; $\mathbf{b}$ subgroup analysis
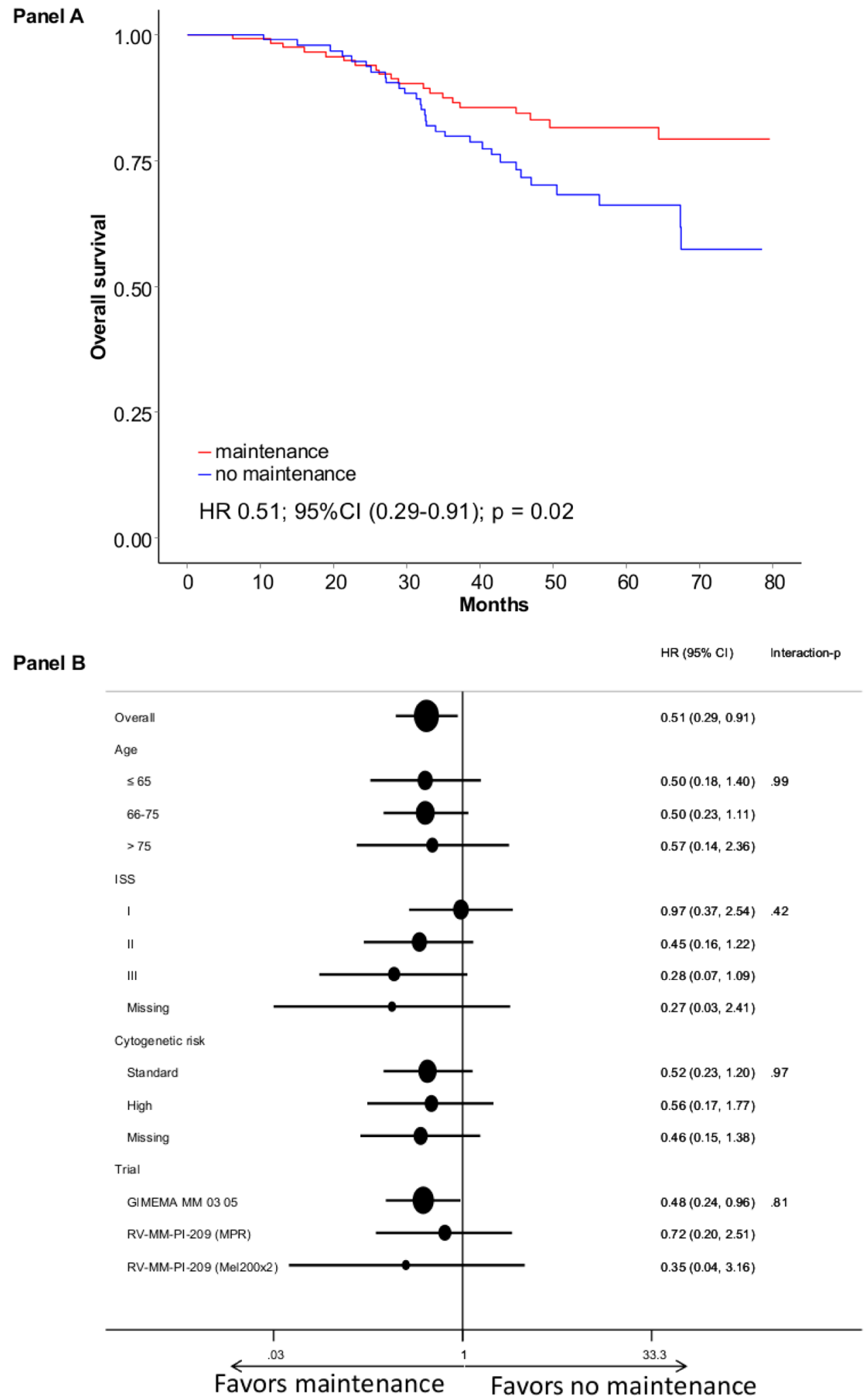

available, to evaluate if maintenance was able to improve the depth of response also in a proportion of CR patients. However, Jackson et al. recently showed that also MRDnegative patients had a PFS benefit with maintenance therapy (Jackson et al. 2016a). Both these findings suggest that prolonged treatment, in patients with chemo-sensitive disease and high-quality responses, is an effective strategy to at least maintain (if not to increase) the depth 

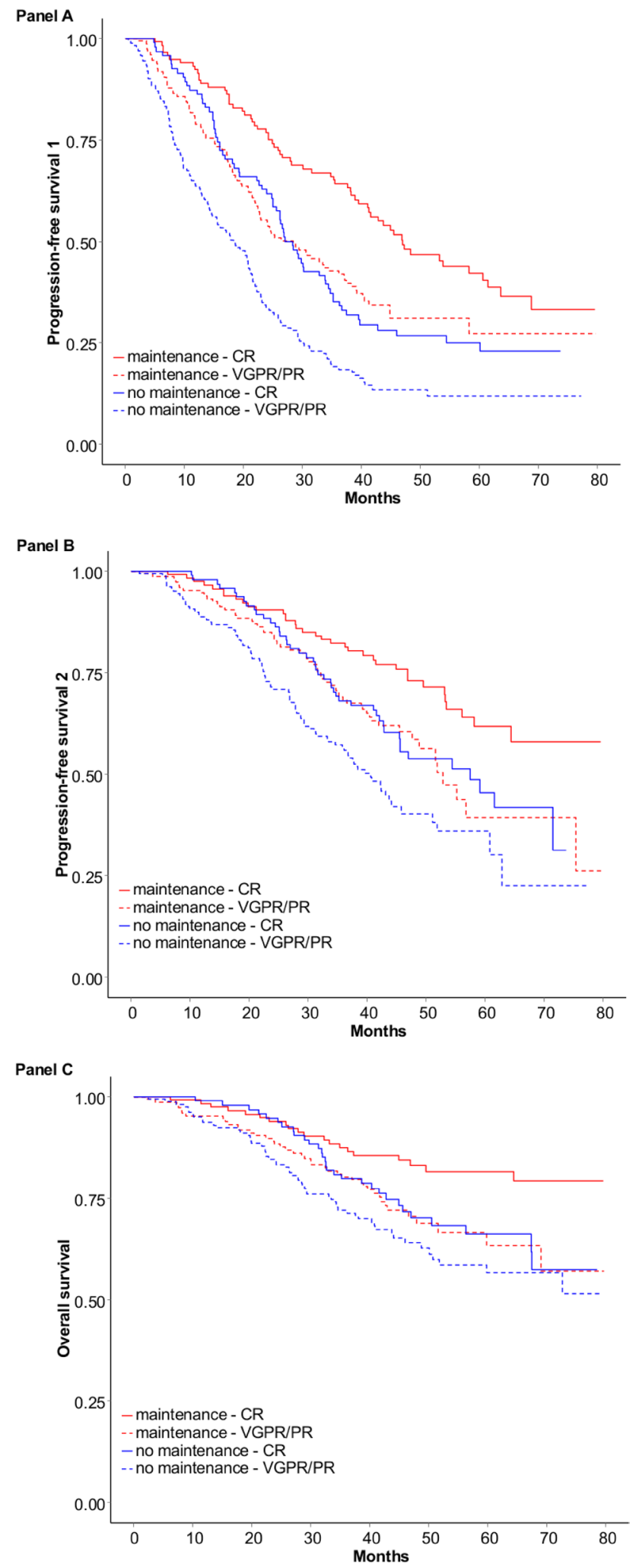

Fig. 4 Survival curves according to best response achieved and maintenance/no maintenance therapy: a progression-free survival 1; b progression-free survival 2; $\mathbf{c}$ overall survival of response obtained during first-line therapy and consequently to improve survival.

In our analyses, the highest survival was observed in CR patients receiving maintenance; survival of patients achieving a $C R$ who did not receive maintenance was similar to the one of VGPR/PR patients treated with maintenance (Fig. 4), as if CR patients not receiving continuous therapy are likely to lose their potential advantage compared with patients obtaining a suboptimal response. Again, similar features were reported by Jackson et al. in MRD-negative patients not receiving maintenance, who showed an outcome similar to MRD patients who received lenalidomide maintenance (Jackson et al. 2016a).

The subgroup analysis showed that the survival advantage for maintenance was retained in all the evaluated subsets of patients. In particular, the benefit derived from maintenance therapy was confirmed in both young and elderly patients; however, the reduction in the risk of progression/death was stronger in patients aged $\leq 65$ and 66-75 years if compared with patients $>75$ years $(p$ of interaction $=0.04)$. Treatment discontinuation rate is commonly higher among elderly patients because of their intrinsic characteristics of frailty. Consequently, the older population has the lowest benefit. In fact, in our study, the rate of discontinuation within 2 years was significantly higher in patients aged $>75$ years, even though the analysis was limited by the low number of patients in this subgroup. A major limitation of this subgroup analysis is that most of patients $<65$ years were enrolled in the RV-MMPI-209 trial, while patients older than 65 were enrolled in the GIMEMA-MM-03-05 trial. The two trials included two different treatment strategies: maintenance with VT was administered for up to 24 months in the GIMEMAMM-03-05; lenalidomide maintenance was administered until progression in the RV-MM-PI-209 (median duration was 23 months). We did see a different impact of maintenance in the two trials ( $p$ of interaction $=0.04$ ). It is not possible to determine if the difference in benefits in young and elderly patients is related to age only, or also to the different treatments administered (VT in elderly, R in young). Nevertheless, a higher rate of discontinuation was observed also among patients aged $>75$ years treated with lenalidomide maintenance (Palumbo et al. 2012).

The analysis has other minor limitations. Data on cytogenetic profile were missing in about $30 \%$ of patients, and ISS data were lacking in $10 \%$ of patients.

In both trials, maintenance randomization was performed at study entry, and not after induction and consolidation. To overcome this limitation, we performed a landmark analysis at 10 months (median duration of induction/consolidation) and we included only patients actually eligible for maintenance. 
Of note, a higher proportion of patients aged $\leq 65$ years obtained less than a CR after induction/consolidation therapy, if compared with elderly patients. Younger patients were mostly enrolled in the RV-MM-PI-209 trial: they received lenalidomide and dexamethasone $(\mathrm{Rd})$ induction and half of them were treated with melphalan-prednisonelenalidomide consolidation, which is now considered a suboptimal induction-consolidation approach (Palumbo et al. 2014b).

PFS2 was not a prespecified objective in the original study protocols. When the date of progression after second-line therapy was not available, the start date of third-line therapy was used to estimate PFS2. Second-line therapies can impact on PFS2 and OS, yet they were not prespecified in both study protocols and they were mostly left to the investigators' discretion.

Maintenance therapy plays an important role in maintaining response and consequently in improving survival in MM. CR, as was defined by IMWG criteria applied at the time of the two trials, is certainly a suboptimal marker of outcome. The IMWG has recently revised response criteria and added new definitions of CR besides the standard categories, according to the new sensitive techniques available to detect MRD (Paiva et al. 2008, 2011, 2012; Kumar et al. 2016). Unfortunately, no MRD data were available at time of analysis, thus it was not possible to evaluate how many CR-MRD positive patients improved to MRD-negative status with maintenance, or to define the impact of maintenance in terms of long-term survival in these patients.

In conclusion, maintenance therapy improved PFS1, PFS2 and OS in MM patients independently of response achieved after induction/consolidation therapy (CR or VGPR/PR), with the highest survival rates with prolonged treatment in patients achieving CR. This could be partly related to a suboptimal definition of $\mathrm{CR}$ if compared with the new response criteria including MRD, even if similar features have been recently seen also in MRD negative patients. However, CR is still the easiest and most widespread method of response evaluation and our data suggest that maintenance therapy should be administered regardless of patient response to maximize outcome. Further studies on the use of MRD will clarify this issue; if the achievement of MRD negativity will imply cure of the disease, maintenance therapy could probably be stopped. Nevertheless, if MRD negativity will be associated with a higher chemo-sensitivity and a subsequent higher burden reduction but not really with cure, maintenance therapy will then remain a good strategy to keep the disease under control.

Prospective randomized trials evaluating a responseadapted approach - possibly including MRD negativity and sustained MRD negativity—are needed to clarify the role of prolonged treatment according to response.
Acknowledgements The authors thank the patients who participated in the source trials, the nurses Manuela Grasso and Luca Merlone, the data managers Jessica Mastrovito and Elena Tigano, the editorial assistants Giorgio Schirripa and Ugo Panzani.

\section{Compliance with ethical standards}

Conflict of interest FDR has received honoraria from Celgene, BMS, and Janssen; FP has received honoraria from Celgene, Janssen, BMS; $\mathrm{CN}$ has served on the Advisory Board for Celgene; TC has received consultancy fees from Takeda and served on the advisory board for Jannsen, Celgene, BMS, Amgen; PM has received Honoraria from Janssen-Cilag and Celgene; $\mathrm{PC}$ has received honoraria from Celgene; MO has received honoraria from Celgene; AP is currently a Takeda employee; MTP has received honoraria from Celgene, Janssen-Cilag, BMS, Amgen, Takeda; MB has received research funding from Amgen, BMS, Celgene, Janssen, Mundipharma, Novartis, Sanofi; and honoraria from AbbVie, Amgen, BMS, Celgene, Janssen, Novartis, Sanofi. FG has served on the advisory board for Takeda, Seattle Genetics, Roche, Mundipharma, Janssen; and received honoraria from Takeda, Amgen, Celgene, Janssen, BMS. All the other authors have no potential conflicts of interest.

Ethical approval GIMEMA-MM-03-05 and RV-MM-PI-209 source studies were approved by the ethics committee of the coordinating site A.O.U. Città della Salute e della Scienza di Torino (Torino, Italy): Comitato Etico Interaziendale A.O.U. Città della Salute e della Scienza di Torino-A.O. Ordine Mauriziano-ASL Città di Torino. All procedures performed in both source studies involving human participants were in accordance with the ethical standards of the institutional and/ or national research committee and with the 1964 Helsinki declaration and its later amendments or comparable ethical standards.

Informed consent Informed consent was obtained from all individual participants included in both source studies.

\section{References}

Altekruse S, Kosary C, Krapcho M et al (2010) SEER cancer statistics review, 1975-2007. In: Natl. Cancer Inst.-Bethesda, MD 2010. https://seer.cancer.gov/archive/csr/1975_2007/. Accessed 31 July 2017

Attal M, Lauwers-Cances V, Marit G et al (2012) Lenalidomide maintenance after stem-cell transplantation for multiple myeloma. $\mathrm{N}$ Engl J Med 366:1782-1791. https://doi.org/10.1056/NEJMoa1114 138

Attal M, Palumbo A, Holstein SA et al (2016) Lenalidomide (LEN) maintenance (MNTC) after high-dose melphalan and autologous stem cell transplant (ASCT) in multiple myeloma (MM): a metaanalysis (MA) of overall survival (OS). J Clin Oncol 34:abstr. 8001

Benboubker L, Dimopoulos MA, Dispenzieri A et al (2014) Lenalidomide and dexamethasone in transplant-ineligible patients with myeloma. N Engl J Med 371:906-917. https://doi.org/10.1056/ NEJMoa1402551

Durie B, Harousseau J-L, Miguel J et al (2006) International uniform response criteria for multiple myeloma. Leukemia 20:1467-1473. https://doi.org/10.1038/sj.leu.2404284

Durie BGM, Hoering A, Abidi MH et al (2017) Bortezomib with lenalidomide and dexamethasone versus lenalidomide and dexamethasone alone in patients with newly diagnosed myeloma without intent for immediate autologous stem-cell transplant 
(SWOG S0777): a randomised, open-label, phase 3 trial. Lancet 389:519-527. https://doi.org/10.1016/S0140-6736(16)31594-X

European Medicines Agency (2012) Appendix 1 to the guideline on the evaluation of anticancer medicinal products in man. Methodological consideration for using progression-free survival (PFS) or disease-free survival (DFS) in confirmatory trials. http://www. ema.europa.eu/docs/en_GB/document_library/Scientific_guide line/2013/01/WC500137126.pdf. Accessed 31 July 2017

Fayers PM, Palumbo A, Hulin C et al (2011) Thalidomide for previously untreated elderly patients with multiple myeloma: metaanalysis of 1685 individual patient data from 6 randomized clinical trials. Blood 118:1239-1247. https://doi.org/10.1182/blood -2011-03-341669

Jackson GH, Davies FE, Pawlyn C et al (2016a) Lenalidomide is a highly effective maintenance therapy in myeloma patients of all ages; results of the phase III myeloma XI study. Blood 128:abst. 1143

Jackson GH, Davies FE, Pawlyn C et al (2016b) Response Adapted induction treatment improves outcomes for myeloma patients; results of the phase III myeloma XI study. Blood 128:abstr. 244

Kumar S, Paiva B, Anderson KC et al (2016) International Myeloma Working Group consensus criteria for response and minimal residual disease assessment in multiple myeloma. Lancet Oncol 17:e328-e346. https://doi.org/10.1016/S1470-2045(16)30206-6

Mateos M-V, Richardson PG, Schlag R et al (2010) Bortezomib plus melphalan and prednisone compared with melphalan and prednisone in previously untreated multiple myeloma: updated follow-up and impact of subsequent therapy in the phase III VISTA trial. J Clin Oncol 28:2259-2266. https://doi.org/10.1200/ JCO.2009.26.0638

Mateos M-V, Oriol A, Martínez-López J et al (2012) Maintenance therapy with bortezomib plus thalidomide or bortezomib plus prednisone in elderly multiple myeloma patients included in the GEM2005MAS65 trial. Blood 120:2581-2588

McCarthy PL, Owzar K, Hofmeister CC et al (2012) Lenalidomide after stem-cell transplantation for multiple myeloma. N Engl J Med 366:1770-1781. https://doi.org/10.1056/NEJMoa1114083

Moreau P, Attal M, Facon T (2015) Frontline therapy of multiple myeloma. Blood 125:3076-3084. https://doi.org/10.1182/blood $-2014-09-568915$

Paiva B, Vidriales M-B, Cerveró J et al (2008) Multiparameter flow cytometric remission is the most relevant prognostic factor for multiple myeloma patients who undergo autologous stem cell transplantation. Blood 112:4017-4023. https://doi.org/10.1182/ blood-2008-05-159624
Paiva B, Martinez-Lopez J, Vidriales M-B et al (2011) Comparison of immunofixation, serum free light chain, and immunophenotyping for response evaluation and prognostication in multiple myeloma. J Clin Oncol 29:1627-1633. https://doi.org/10.1200/ JCO.2010.33.1967

Paiva B, Gutiérrez NC, Rosiñol L et al (2012) High-risk cytogenetics and persistent minimal residual disease by multiparameter flow cytometry predict unsustained complete response after autologous stem cell transplantation in multiple myeloma. Blood 119:687691. https://doi.org/10.1182/blood-2011-07-370460

Palumbo A, Anderson K (2011) Multiple myeloma. N Engl J Med 364:1046-1060. https://doi.org/10.1056/NEJMra1011442

Palumbo A, Bringhen S, Rossi D et al (2010) Bortezomib-melphalan-prednisone-thalidomide followed by maintenance with bortezomib-thalidomide compared with bortezomib-melphalan-prednisone for initial treatment of multiple myeloma: a randomized controlled trial. J Clin Oncol 28:5101-5109. https://doi. org/10.1200/JCO.2010.29.8216

Palumbo A, Waage A, Hulin C et al (2012) Safety of thalidomide in newly diagnosed elderly myeloma patients: a meta-analysis of data from individual patients in six randomized trials. Haematologica 98:87-94. https://doi.org/10.3324/haematol.2012.067058

Palumbo A, Bringhen S, Larocca A et al (2014a) Bortezomib-melphalan-prednisone-thalidomide followed by maintenance with bortezomib-thalidomide compared with bortezomib-melphalan-prednisone for initial treatment of multiple myeloma: updated follow-up and improved survival. J Clin Oncol 32:634-640. https ://doi.org/10.1200/JCO.2013.52.0023

Palumbo A, Cavallo F, Gay F et al (2014b) Autologous transplantation and maintenance therapy in multiple myeloma. N Engl J Med 371:895-905. https://doi.org/10.1056/NEJMoa1402888

Palumbo A, Gay F, Cavallo F et al (2015) Continuous therapy versus fixed duration of therapy in patients with newly diagnosed multiple myeloma. J Clin Oncol 33:3459-3466. https://doi. org/10.1200/JCO.2014.60.2466

San Miguel JF, Schlag R, Khuageva NK et al (2008) Bortezomib plus melphalan and prednisone for initial treatment of multiple myeloma. N Engl J Med 359:906-917. https://doi.org/10.1056/ NEJMoa0801479

van de Velde HJK, Liu X, Chen G et al (2007) Complete response correlates with long-term survival and progression-free survival in high-dose therapy in multiple myeloma. Haematologica 92:13991406. https://doi.org/10.3324/haematol.11534

\section{Affiliations}

\section{Chiara Cerrato ${ }^{1}$. Francesco Di Raimondo ${ }^{2} \cdot$ Lorenzo De Paoli $^{3} \cdot$ Stefano Spada ${ }^{1} \cdot$ Francesca Patriarca $^{4}$. Claudia Crippa ${ }^{5}$. Roberto Mina ${ }^{1} \cdot$ Tommasina Guglielmelli $^{6}$. Dina Ben-Yehuda ${ }^{7}$. Daniela Oddolo ${ }^{1}$. Chiara Nozzoli ${ }^{8}$. Emanuele Angelucci ${ }^{9}$. Nicola Cascavilla ${ }^{10}$ - Rita Rizzi ${ }^{11}$ - Stefano Rocco ${ }^{12}$. Luca Baldini $^{13}$ - Elena Ponticelli ${ }^{1}$. Magda Marcatti ${ }^{14}$. Clotilde Cangialosi ${ }^{15} \cdot$ Tommaso Caravita $^{16}$. Giulia Benevolo ${ }^{17}$. Roberto Ria ${ }^{18}$. Arnon Nagler ${ }^{19}$. Pellegrino Musto ${ }^{20} \cdot$ Paola Tacchetti $^{21} \cdot$ Paolo Corradini $^{22} \cdot$ Massimo Offidani $^{23} \cdot$ Antonio Palumbo $^{1,25}$. Maria Teresa Petrucci ${ }^{24} \cdot$ Mario Boccadoro ${ }^{1} \cdot$ Francesca Gay $^{1}[$}

1 Myeloma Unit, Division of Hematology, University of Torino, Azienda-Ospedaliero Universitaria Città della Salute e della Scienza di Torino, via Genova 3, 10126 Torino, Italy

2 UOC Ematologia, Azienda Policlinico-OVE, University of Catania, Catania, Italy
3 Division of Hematology, Department of Translational Medicine, University of Eastern Piedmont and Maggiore Hospital, Novara, Italy

4 Dipartimento di Medicina, Università di Udine, Udine, Italy

5 Divisione di Ematologia, Spedali Civili Brescia, Brescia, Italy 
6 Department of Clinical and Biological Sciences, Orbassano, TO, Italy

7 Hematology Department, Hadassah Medical Center, Jerusalem, Israel

8 SODc Terapie cellulari e medicina trasfusionale AOUC Careggi, Firenze, Italy

9 IRCCS Azienda Ospedaliera Universitaria San Martino - IST Istituto Nazionale per la Ricerca sul Cancro, Genova, Italy

10 Hematology "Casa Sollievo della Sofferenza" Hospital IRCCS, San Giovanni Rotondo, FG, Italy

11 Section of Hematology with Transplantation, Department of Emergency and Organ Transplantation, University of Bari Medical School, Bari, Italy

12 UOSC Ematologia con Trapianto - AORN Cardarelli Napoli, Napoli, Italy

13 Department of Oncology and Hemato-oncology, University of Milan, Fondazione IRCCS Cà Granda, Ospedale Maggiore Policlinico, Milano, Italy

14 U.O. Ematologia e Trapianto di Midollo, IRCCS Ospedale San Raffaele, Milano, Italy

15 Ospedali Riuniti Villa Sofia-Cervello, Unità Operativa di Ematologia I e UTMO, Palermo, Italy
16 UOC Ematologia Ospedale S. Eugenio ASL RM2, Roma, Italy

17 SC Hematology, Città della Salute e della Scienza Hospital, Torino, Italy

18 University of Bari "Aldo Moro" Medical School, Department of Biomedical Science, Internal Medicine "G. Baccelli", Policlinico, Bari, Italy

19 Hematology Division, Chaim Sheba Medical Center, Tel HaShomer, Ramat Gan, Israel

20 Scientific Direction, IRCCS Referral Cancer Center of Basilicata, Rionero in Vulture, PZ, Italy

21 “Seràgnoli” Institute of Hematology, Department of Experimental, Diagnostic and Specialty Medicine, Bologna University School of Medicine, Bologna, Italy

22 Department of Oncology and Hematology, University of Milano, Milano, Italy

23 Clinica di Ematologia, AOU Ospedali Riuniti di Ancona, Loc. Torrette, Ancona, Italy

24 Hematology, "Sapienza" University of Rome, Roma, Italy

25 Takeda, Zürich, Switzerland 